Title: Intervention development for exercise promotion at active charity events in the United Kingdom.

Authors: ${ }^{1,2}$ Bethany Alice Jones ${ }^{*},{ }^{1,2}$ Fehmidah Munir, ${ }^{3}$ Philine S Harris, ${ }^{4}$ Prachi Bhatnagar, ${ }^{1,2}$ Clare Stevinson

${ }^{1}$ School of Sport, Exercise and Health Sciences, Loughborough University

${ }^{2}$ National Centre of Sport and Exercise Medicine East Midlands

${ }^{3}$ Centre for Clinical and Community Applications of Health Psychology, Department of Psychology, University of Southampton

${ }^{4}$ Centre on Population Approaches for Non-Communicable Disease Prevention, Nuffield Department of Population Health, University of Oxford

*Current affiliation is School of Social Sciences, Department of Psychology, Nottingham Trent University

Acknowledgments: We would like to acknowledge the support of Helen Curtis, Shelly Kassam, and Karen Milton in contributing to the project planning group, and Lorna Hartley in facilitating data collection at a Race for Life event.

Funding statement: This work was supported by a cancer prevention innovation award from the Cancer Research UK Population Research Committee (grant number C7647/A25892).

Ethical approval: The study was reviewed and approved by Loughborough University Ethics Approvals (Human Participants) Sub-Committee.

Key words: physical activity, intervention mapping 


\title{
Intervention development for exercise promotion at active charity events in the United
}

\section{Kingdom.}

\begin{abstract}
Background: This study used the Intervention Mapping protocol to design an evidence-based intervention package for organisers of active charity events to support their participants in remaining or becoming regular exercisers.
\end{abstract}

Methods: A mixed-methods approach following the Intervention Mapping protocol was used to develop intervention components. A needs assessment was initially performed to identify the behavioural and environmental determinants of exercise for charity event participants (step 1). Next, the intended intervention outcomes, and performance and change objectives were specified (step 2). Theory-based change methods were selected and matched with practical strategies (step 3). This resulted in the design of the first iteration of the intervention which underwent pre-testing with former event participants and feasibility testing at an active charity event (step 4).

Results: The evidence-based interventions included components to implement at events (e.g., an activity and information zone, and exercise planner), along with elements pre- and postevent (e.g., social media). Pre-testing indicated high acceptability of the planned components, but feasibility testing suggested low engagement with the intervention.

Conclusion: Despite developing the intervention package through the systematic process of Intervention Mapping, preliminary data suggest that further development and testing is needed to refine the intervention before implementation. 


\section{INTRODUCTION}

Physical inactivity is one of the leading modifiable risk factors for ill-health estimated to account for $9 \%$ of premature mortality worldwide (Lee et al, 2012). The World Health Organisation (2010) recommends a minimum of 150 minutes per week of moderate intensity physical activity or 75 minutes of vigorous exercise for health maintenance in adults. In the United Kingdom self-reported physical activity data suggest that $42 \%$ of women and $34 \%$ of men are below this threshold (National Statistics, 2018), and population-wide physical activity promotion has become a national priority in recent years (Department of Health, 2011).

Community-based activity schemes have been highlighted as potentially cost-effective approaches to exercise promotion (Pringle et al, 2009), and are recommended for their ability to influence social norms for health and exercise (Department of Health, 2011). In particular, cause-based fundraising events involving physical activity (e.g. charity bicycle rides and fun runs) have been identified as a way of indirectly engaging low-active individuals in physical activity though charitable and social motives (Robinson, 2010). Since events often encourage participation at low levels of exercise intensity (e.g. walking) in a non-competitive atmosphere they are inclusive of those who are not regular exercisers (Bauman et al, 2009).

Such events have considerable reach attracting high volumes of people with varying levels of fitness and reasons for participation. A study of 494 college students identified philanthropy and desire to support the charity as the strongest motives for taking part in charity sports events, particularly among females. However, sporting and social reasons were also prevalent (Won \& Park, 2010). In another study involving 579 participants of active fundraising events, connection to the charity was the dominant reason for taking part. However, desire to pursue a healthy lifestyle was also identified as a strong motive, suggesting that participants would be receptive to exercise promotion interventions (Bennett et al, 2007).

Previous interventions in this context have involved educational materials. Women who had taken part in a mass participation $10 \mathrm{~km}$ event in Ireland were randomly allocated to receive either a printed booklet tailored to motivational stage of readiness for exercise or a standardised healthy eating booklet (Lane et al, 2010). After 6 months, physical activity had increased to a similar degree in both conditions suggesting that the tailored materials had no specific influence, but that participants responded to the informational prompt. Notably the 
materials were modified versions of those used in an earlier study in Australia, rather than being specifically designed for the population and setting.

One systematic and effective approach to intervention development is Intervention Mapping, a six-step iterative process specifically intended for health promotion (Bartholomew et al, 2016). The Intervention Mapping protocol acknowledges both personal and environmental determinants of behaviour and ensures that interventions are grounded in theory (Brug et al, 2005). This approach has previously been used to design successful physical activity promotion interventions (Gillison et al, 2012; McEachan et al 2008).

This study employed the Intervention Mapping protocol to design a scalable intervention package that can be implemented by charities delivering physically active fundraising events, in order to support their participants in maintaining or adopting regular exercise. It focused specifically on Race for Life, a national series of annual women-only non-competitive $5 \mathrm{~km}$ or $10 \mathrm{~km}$ runs/walks, organised by Cancer Research UK. Over 500,000 women take part across more than 300 Race for Life events each summer, contributing to its status as the biggest fundraising campaign in the UK (The Civil Society, 2017; Third Force News, 2015). Previous data based on 228 women taking part in Race for Life (McVinnie et al, 2017) indicated that nearly a quarter $(22 \%)$ of participants reported being typically inactive or low active, and a large proportion (47\%) were overweight or obese. Notably, women who walked the event (36\%) had significantly lower levels of self-efficacy and intrinsic motivation for exercise than women who ran or jogged. The Race for Life population was therefore recognised to have potential for benefit from an intervention aimed at promoting maintenance or adoption of regular exercise. If the intervention package developed here ultimately proves effective after implementation and evaluation, continued research using the Intervention Mapping protocol will be undertaken to adapt it to the different settings and populations of other active fundraising events.

\section{METHOD}

A planning group was established to guide the Intervention Mapping process involving Race for Life staff and participants, members of the research team, and an independent academic. 


\section{Step 1. Needs assessment}

The first step of the process was to assess the behavioural and environmental determinants of exercise after active charity events. The needs assessment involved a rapid review of relevant literature, and interviews with Race for Life organisers and participants.

For the rapid review, literature concerned with mass participation charity events and reviews of interventions for promoting physical activity were located through searching four electronic databases (Pubmed; SportDiscus; PsychInfo; Web of Science). To locate literature on mass participation charity events, terms relating to physical activity (e.g. sport*, walk*, run*, cycl*, exercis*, activ*) were combined with search terms related to charity (e.g. charit*, fundrais*). These searches returned 996 results and after screening, 25 articles were included (supplementary file). For the search of reviews of interventions for promoting physical activity, search terms included physical activ*, exercis*, intervention*, and the search was restricted to reviews. This search returned 591 articles with 34 retained following screening (supplementary file).

Four employees of Cancer Research UK who were involved with Race for Life or other active fundraising events took part in semi-structured telephone interviews to explore approaches that may be effective in this context to support regular physical activity. Interviews were also undertaken with 20 women who had previously taken part in a Race for Life event to explore motives for signing up and perceived outcomes of participation, along with barriers and motivation relating to exercise outside of active charity events. Interviews were recorded, transcribed verbatim, and systematically coded to identify common themes (Braun et al, 2016).

\section{Step 2. Identification of intended outcomes and performance objectives}

The second step of the process involved identifying the expected behavioural and environmental outcomes from the intervention in the target population and defining performance objectives for these outcomes. Performance objectives include a description of what is required of the target population to perform the behavioural outcome, or how environmental conditions will be modified. This led to the creation of a matrix of change in which the change objectives are specified. The matrix combines the evidence-and theorybased determinants of the behavioural and environmental outcomes that were identified as part of the needs assessment with the performance objectives. Change objectives indicate 
what needs to change in the determinant to affect the performance objective and represent the most immediate targets of an intervention.

\section{Step 3. Programme design}

Step 3 involved designing the intervention under the guidance of the planning group. Theoryand evidence-based change methods were selected on the basis that they could address the change objectives from step 2. This involved identifying approaches appropriate for influencing the determinant for each of the specific change objectives and considering the known parameters for effectiveness. Ideas for the intervention identity were also generated as well as discussing the components of the intervention and delivery schedules.

\section{Step 4. Programme production}

In step 4 the intervention materials and protocols were generated based on the designs agreed with the planning group. Pre-testing of all the provisional intervention components involved six former Race for Life participants who accessed electronic versions of each intervention component and provided ratings and free-text feedback on acceptability via an online questionnaire.

Pilot-testing was undertaken at a Race for Life event to examine the feasibility of delivering the intervention package and participant engagement. Adult women who had completed the $5 \mathrm{~km}$ event were invited to view materials and complete a short questionnaire on the day of the event. This included a measure of recent exercise based on the Godin Leisure-Time Exercise Questionnaire (Godin and Shephard, 1985). Participants recorded the frequency and duration of light, moderate and strenuous exercise sessions performed during leisure time across a typical week in the past month. They also rated five determinants of exercise from 0 (not at all) to 10 (completely) to indicate the extent of their: (1) confidence; (2) motivation; (3) social support, (4) knowledge, and (5) ability to plan exercise. Email addresses were requested so that follow-up questionnaires about engagement with the intervention and exercise levels could be sent one month after the Race for Life event. Participants were given all materials to keep and were told that for each completed follow-up questionnaire received, a small donation (1GBP) would be made to Cancer Research UK.

\section{Steps 5 and 6. Programme implementation and evaluation plans}


From the start of the intervention planning process, both implementation of the intervention and evaluation should be considered. If, after step 4, the intervention is ready for adoption, a detailed plan for implementation should be finalised as well as a matrix of change objectives based on outcome and performance objectives for programme adoption, implementation and maintenance (step 5). The evaluation plan should also be finalised (step 6) and this should include both assessments of effect (effectiveness of the intervention) and process (delivery of the intervention).

\section{RESULTS}

\section{Step 1. Needs assessment}

Evidence for mass participation charity events: Common reasons for taking part in mass charity events included wanting to: support a good cause, experience a sense of camaraderie, feel connected to the event, increase physical and mental health, and undertake a personal challenge (Bixler, 2014; Filo et al, 2008, 2009, 2010, 2011, 2014; Filo et al, 2013, Jeffery, 2012; Rundio et al, 2014; Scott and Solomon, 2003; Snelgrove et al, 2013; Won and Park, 2010). Similarly, outcomes reported from event participation were feeling an attachment to the charity (Filo et al, 2010; Filo et al, 2012; Snelgrove et al, 2013), camaraderie (Filo et al, 2008; Filo et al, 2013), positive wellbeing (e.g., joy) and a sense of achievement (Filo and Coghlan, 2016).

Reviews of interventions for promoting physical activity: Interventions that were underpinned by behavioural theories were found to be better designed and more effective than atheoretical interventions (McIntosh et al, 2017). Common evidence-based behavioural techniques employed were: goal setting, self-monitoring, follow-up prompts, barrier identification, problem solving, and motivational interviewing (Avery et al, 2012; Avery et al, 2015; French et al, 2014; Fjeldsoe et al, 2011; George et al, 2012; Greaves et al, 2011; Middelweerd et al, 2014; Murray et al, 2017; Samdal et al, 2017). Multi-component approaches tended to be most effective (Fjeldsoe et al, 2011; George et al, 2012; Goode et al, 2012; Greaves et al, 2011; Jenkins, 2009; Richards et al, 2013; Schoeppe et al, 2016), and electronic delivery methods were well supported (Hakala et al, 2017; Oosterveen et al, 2017). Finally, interventions involving social media were identified as useful for self-monitoring, observing others engage in exercise, accessing motivating quotes, and creating social connectedness, although overall effectiveness on physical activity behaviour was mixed (Maher et al, 2014, McIntosh, , 2017; Laranjo et al, 2014; Williams et al, 2014). 
Interviews with event organisers and participants: Analysis of the four interviews with the Cancer Research UK employees identified several strategies to consider for the intervention. These included the provision of a training plan in advance of the charity event to instil a pattern of regular exercise motivated by the goal of race completion. Other suggestions involved encouraging participants on the day of the event to make future exercise commitments or pledges and to sign up to local exercise opportunities. Ways of consolidating the sense of achievement from taking part through providing a memory of the day (e.g. a finisher's photograph) were discussed along with the importance of promoting health and well-being benefits of exercise. For the interviews with event participants, a summary of findings relating to motives and outcomes of taking part in Race for Life, along with barriers and facilitators to exercise outside of the charity event is provided in Table 1.

\section{Step 2. Identification of intended outcomes and performance objectives}

The overall expected behavioural outcomes from this intervention were the maintenance, increase or adoption of regular exercise among charity event participants. The expected environmental outcomes were: support from friends and family to exercise regularly, identification of exercise role models (interpersonal environment), and awareness of difference exercise opportunities (community environment). Next, performance objectives were developed by listing the steps that were required to achieve the intervention outcomes. The needs assessment identified several determinants from Social Cognitive Theory (Bandura, 1986) as relevant for this intervention (self-efficacy, social support, outcome expectations and expectancies, knowledge and self-regulation). The matrix of change was developed by crossing the performance objectives with the determinants (Table 2).

\section{Step 3. Programme design}

Theory and evidence-based change methods that would address the change objectives were selected. This list of change methods was presented to the planning group and refined. The change methods selected are presented in Table 3 along with the parameters for effectiveness and the practical strategies that were agreed by the planning group. Discussions around programme identity led to the name "Good Start" for the intervention package.

\section{Step 4. Programme production}


The first draft of the intervention materials that incorporated the practical strategies outlined in step 3 were designed incorporating five related components as outlined below.

Postcard: The postcard was given to participants after crossing the finish line. Based on selfaffirmation principles, the postcard encouraged participants to identify their positive feelings after completing the event (e.g. pride, happiness) through a checklist and to reflect on these in the future to stimulate exercise motivation.

Good Start zone: At the Race for Life event the Good Start zone consisted of a coloured gazebo and a large Good Start flag displaying the logo. Within the zone, exercise equipment was available with Good Start helpers to introduce participants to some taster activities (e.g. hula hoop, mini-trampoline, lolo ball), as examples of low-cost forms of exercise not requiring space or facilities. A poster was displayed within the zone that included different exercise opportunities to remind participants of the range of options available. Materials for participants to take away included educational puzzles conveying information on exercise benefits and lists of nationwide free or low-cost exercise opportunities. Copies of the Good Start exercise guide (described below) were also available to participants in the zone.

Good Start exercise guide: The Good Start guide included exercise and health information, advice and encouragement. Space was provided to develop weekly exercise goals and make action plans relating to exercise. Four weeks of planners were included within the guide, with a weblink provided through which more planners could be downloaded. Exercise tips, images, and quotations from former Race for Life participants were incorporated within the guide.

Social media: An Instagram page dedicated to the intervention was developed including photographs with motivating exercise quotes, Race for Life participant quotes, and exercise hints and tips. A hashtag was also generated through which Race for Life participants could share their exercise journey by using the hashtag on Instagram.

Pre-event and post-event emails: E-mails were planned for participants before and after their Race for Life event. Pre-event this included an introduction to the Good Start project, and an invitation to visit the Instagram page, and to visit the Good Start zone at the event. The list of suggested exercise opportunities was also linked to assist participants in preparing for the 
$5 \mathrm{~km}$ event. Post-event the e-mail congratulated participants on completing the $5 \mathrm{~km}$ and repeated the invitation to visit the Instagram page for support in maintaining exercise. There was also a link to the list of exercise opportunities and to further exercise planners available to download.

Pre-testing: Overall, the intervention components were rated highly by the pre-testing sample $(n=6)$. Based on their feedback more content and images were added to the Instagram page and Good Start guide to ensure that they were relevant to women of all ages and fitness levels.

Pilot testing: In total 97 women completed the questionnaire, with a median age of 41 years (interquartile range: 27-51). Participants reported a median of 100 minutes per week (interquartile range: 23-172) of moderate-strenuous exercise in the past month and 30 minutes (interquartile range: 0-120) of light exercise. Just over one third of the sample (34.0\%) was meeting the public health guideline of $\geq 150$ minutes per week of moderatevigorous physical activity. Median ratings (out of 10) were high for all exercise determinants: confidence (7), motivation (7), support (7), knowledge (8), planning (6).

Fourteen participants did not provide their email address and a further 15 addresses were invalid, hence 69 people were emailed the follow-up questionnaire one month later of which 15 completed the questionnaire ( $22 \%$ response rate). The majority of participants completed the postcard after the Race for Life event $(n=12)$. However, only two reported reflecting on their responses in the following weeks. The components of the Good Start exercise guide were rated as useful by most participants: exerciser planners $(n=9)$, goal-setting $(n=9)$, exercise tips $(n=11)$, health information $(n=10)$, participant quotes $(n=13)$, exercise opportunities $(n=10)$, except for the educational puzzles $(n=5)$. Of eleven participants who reported visiting the Instagram page, eight rated the content as slightly motivating, and two as very motivating. None of the participants reported visiting the Good Start zone due to not realising the zone existed $(n=11)$, being too hot and tired $(n=2)$ or having insufficient time to visit $(\mathrm{n}=2)$.

A comparison of the exercise levels reported by the 15 women at baseline and 1-month follow-up indicated no significant group-level changes for moderate-vigorous exercise or light exercise through Wilcoxon signed-rank tests $(p>.05)$. Individual participant data 
revealed mixed patterns of change. Among six women who were meeting physical activity guidelines at baseline, three continued to do so at one month, and three others had dropped below guideline levels. Of the nine who were insufficiently active at baseline, four had increased exercise to meet the guidelines. There were also no significant changes observed in the ratings of exercise determinants and an inconsistent pattern of small increases and decreases across the sample.

Although implementation (step 5) and evaluation (step 6) plans had been considered from the outset of the project the planning group agreed that the preliminary testing results were not sufficient to recommend implementation or evaluation given the low response rate to the follow up questionnaire, and limited engagement with the intervention components. Instead it was proposed that earlier stages of the Intervention Mapping process were revisited before more comprehensive pre- and pilot-testing.

\section{DISCUSSION}

This study used the Intervention Mapping protocol to develop an intervention package that could be implemented in relation to Race for Life fundraising events, to support maintenance or adoption of regular exercise. The intervention components designed through this systematic process were evidence-based and tailored to the needs of charity event participants.

Despite positive indications of acceptability from pre-testing, there was low engagement with most intervention components during pilot testing. This made it difficult to interpret the potential value of the intervention for future evaluation, with no consistent patterns of maintenance or change in self-reported exercise one-month after the event, and no change in the core determinants of exercise on which the intervention was based.

This study supports existing evidence that exercise is a challenging behaviour to change in any setting (Marcus et al, 2000; Marcus et al, 2006). Reviews of physical activity change interventions suggest that the most promising approaches are comprehensive multicomponent individualised programmes with ongoing booster strategies (MüllerRiemenschneider et al, 2008). However, provision of intensive schemes can be limited by cost and available personnel for delivery, and the long-term sustainability of behaviour has not been established (Müller-Riemenschneider et al, 2008). Similarly, community-wide 
interventions have had limited success in bringing measurable change in physical activity in the populations studied (Baker et al, 2015). The need for effective approaches that can be implemented on a large scale remains strong.

A strong theoretical basis is recommended for behaviour change interventions, and this is a core part of the Intervention Mapping process. Constructs from Social Cognitive Theory (Bandura, 1986) emerged as important from the needs assessment and were used in the design of the intervention components. This theory is one of the most widely used in physical activity intervention research (Marcus et al., 2006), and self-efficacy in particular has been identified as a consistent predictor of physical activity across the lifespan (Bauman et al., 2012). A meta-analysis of 27 studies in healthy adults suggested that interventions based on self-efficacy led to small but significant changes in both underlying self-efficacy and physical activity behaviour (Williams and French, 2011). Some of the strongest results were for goalspecific action planning interventions, which was one of the key features of the Good Start exercise guide. Nonetheless, despite theoretical and empirical evidence supporting the key components of the Good Start intervention, engagement was low hence the importance of revisiting earlier steps of the Intervention Mapping protocol.

A major strength the Intervention Mapping approach is the flexibility to return to earlier stages of the process to ensure the change objectives and methods are appropriately targeted for the population and the intervention design is robust. The current study recruited small samples for the needs assessment as well as for pre and pilot testing. However, there may be value in including larger samples across the diverse target population to help identify a broader range of exercise determinants and improve the intervention design.

\section{Conclusion}

This study followed the Intervention Mapping protocol to develop a theoretically based intervention package aimed at supporting participants of a physically active fundraising event in maintaining or adopting regular exercise beyond the event. Preliminary data did not suggest proceeding to the steps of implementation and evaluation, and further development and testing is required to refine the intervention. 


\section{References}

Avery, L., Flynn, D., Van Wersch, A., Sniehotta, F. F., \& Trenell, M. I. (2012). Changing physical activity behavior in type 2 diabetes: a systematic review and meta-analysis of behavioral interventions. Diabetes Care, 35(12), 2681-2689. doi: /10.2337/dc11-2452

Avery, L., Flynn, D., Dombrowski, S. U., van Wersch, A., Sniehotta, F. F., \& Trenell, M. I. (2015). Successful behavioural strategies to increase physical activity and improve glucose control in adults with Type 2 diabetes. Diabetic Medicine, 32(8), 1058-1062. doi:/10.1111/dme.12738

Baker, P.R.A., Francis, D.P., Soares, J., Weightman, A.L., Foster, C. (2015) Community wide interventions for increasing physical activity. Cochrane Database of Systematic Reviews;1:CD008366. doi: 10.1002/14651858.CD008366.pub3

Bandura, A. (1986). Social foundations of thought and action: A social cognition theory. Englewood Cliffs, NJ: Prentice-Hall.

Bartholomew Eldredge, L. K., Markham, C. M., Ruiter, R. A., Fernández, M. E., Kok, G., \& Parcel, G. S. (2016). Planning health promotion programs: An intervention mapping approach. San Francisco: Jossey-Bass.

Bauman, A., Murphy, N., \& Lane, A. (2009). The role of community programmes and mass events in promoting physical activity to patients. British Journal of Sports Medicine, 43(1), 44-46. doi:10.1136/bjsm.2008.054189.

Bennett, R., Mousley, W., Kitchin, P., \& Ali-Choudhury, R. (2007). Motivations for participating in charity-affiliated sporting events. Journal of Customer Behaviour, 6(2), 155-178. doi: 10.1362/147539207X223375

Bixler, N. R. (2014). How a weekend becomes an ideal: pilgrimage in the breast cancer walk. Leisure Studies, 33(2), 133-147. doi: 10.1080/02614367.2013.833285

Braun, V., Clarke, V., \& Weate, P. (2016) Using thematic analysis in sport and exercise research. In Smith B, Sparks A. (Eds.) Routledge Handbook of Qualitative Research Methods in Sport and Exercise (pp. 191-205). London: Routledge.

Brug, J., Oenema, A., \& Ferreira, I. (2005). Theory, evidence and Intervention Mapping to improve behavior nutrition and physical activity interventions. International Journal of Behavioral Nutrition and Physical Activity, 2(1), 2. doi:10.1186/1479-5868-2-2

Department of Health. (2011). Start Active, Stay Active - a report on physical activity for health from the four home countries' Chief Medical Officers. London: Department of Health, 2011. 
Filo, K., Funk, D. C., \& O’Brien, D. (2008). It's really not about the bike: Exploring attraction and attachment to the events of the Lance Armstrong Foundation. Journal of Sport Management, 22, 501-525.

Filo, K., Funk, D. C., \& O’Brien, D. (2009). The meaning behind attachment: Exploring camaraderie, cause, and competency at a charity sport event. Journal of Sport Management, 23(3), 361-387. doi: 10.1123/jsm.23.3.361

Filo, K., Funk, D., \& O’Brien, D. (2010). The antecedents and outcomes of attachment and sponsor image within charity sport events. Journal of Sport Management, 24(6), 623648. doi:10.1123/jsm.24.6.623

Filo, K., Funk, D. C., \& O'Brien, D. (2011). Examining motivation for charity sport event participation: A comparison of recreation-based and charity-based motives. Journal of Leisure Research, 43(4), 491-518. doi:10.1080/00222216.2011.11950247

Filo, K., Groza, M. D., \& Fairley, S. (2012). The role of belief in making a difference in enhancing attachment to a charity sport event. Journal of Nonprofit \& Public Sector Marketing, 24(2), 123-140. doi: 10.1080/10495142.2012.679165

Filo, K., Funk, D. C., \& O'Brien, D. (2014). An empirical investigation of the role of camaraderie, cause, competency, and participation motives in the development of attachment to a charity sport event. Managing Leisure, 19(4), 245-262. doi:10.1080/13606719.2014.885715

Filo, K., Spence, K., \& Sparvero, E. (2013). Exploring the properties of community among charity sport event participants. Managing Leisure, 18(3), 194-212. doi: $10.1080 / 13606719.2013 .796179$

Filo, K., \& Coghlan, A. (2016). Exploring the positive psychology domains of well-being activated through charity sport event experiences. Event Management, 20(2), 181-199. doi: /10.3727/152599516X14610017108701

Fjeldsoe, B., Neuhaus, M., Winkler, E., \& Eakin, E. (2011). Systematic review of maintenance of behavior change following physical activity and dietary interventions. Health Psychology, 30(1), 99. doi: 10.1037/a0021974

French, D. P., Olander, E. K., Chisholm, A., \& McSharry, J. (2014). Which behaviour change techniques are most effective at increasing older adults' self-efficacy and physical activity behaviour? A systematic review. Annals of Behavioral Medicine, 48(2), 225234. doi: /10.1007/s12160-014-9593-Z

George, E. S., Kolt, G. S., Duncan, M. J., Caperchione, C. M., Mummery, W. K., Vandelanotte, C., ... \& Noakes, M. (2012). A review of the effectiveness of physical 
activity interventions for adult males. Sports Medicine, 42(4), 281-300. Doi:

$10.2165 / 11597220-000000000-00000$

Gillison, F., Greaves, C., Stathi, A., Ramsay, R., Bennett, P., Taylor, G., Francis, M., \& Chandler, R. (2012). 'Waste the Waist': the development of an intervention to promote changes in diet and physical activity for people with high cardiovascular risk. British Journal of Health Psychology, 17(2), 327-345. doi:10.1111/j.2044-8287.2011.02040.x

Goode, A. D., Reeves, M. M., \& Eakin, E. G. (2012). Telephone-delivered interventions for physical activity and dietary behavior change: an updated systematic review. American Journal of Preventive Medicine, 42(1), 81-88. doi:/10.1016/j.amepre.2011.08.025

Godin, G., \& Shephard, R. J. (1985). A simple method to assess exercise behavior in the community. Canadian Journal of Applied Sport Sciences, 10(3), 141-146.

Greaves, C. J., Sheppard, K. E., Abraham, C., Hardeman, W., Roden, M., Evans, P. H., \& Schwarz, P. (2011). Systematic review of reviews of intervention components associated with increased effectiveness in dietary and physical activity interventions. BMC public health, 11(1), 119. doi:/10.1186/1471-2458-11-119

Hakala, S., Rintala, A., Immonen, J., Karvanen, J., Heinonen, A., \& Sjögren, T. (2017). Effectiveness of technology-based distance interventions promoting physical activity: systematic review, meta-analysis and meta-regression. Journal of Rehabilitation Medicine, 49(2), 97-105. doi:/10.2340/16501977-2195

Jeffery, K. A., \& Butryn, T. K. (2012). The motivations of runners in a cause-based marathon-training program. Journal of Sport Behavior, 35(3), 300.

Jenkins, A., Christensen, H., Walker, J. G., \& Dear, K. (2009). The effectiveness of distance interventions for increasing physical activity: a review. American Journal of Health Promotion, 24(2), 102-117. doi:/10.4278/ajhp.0801158

Lane, A., Murphy, N., Bauman, A., \& Chey, T. (2010). Randomized controlled trial to increase physical activity among insufficiently active women following their participation in a mass event. Health Education Journal, 69(3), 287-296. doi: $10.1177 / 0017896910364890$

Laranjo, L., Arguel, A., Neves, A. L., Gallagher, A. M., Kaplan, R., Mortimer, N., ... \& Lau, A. Y. (2014). The influence of social networking sites on health behavior change: a systematic review and meta-analysis. Journal of the American Medical Informatics Association, 22(1), 243-256. doi: 10.1136/amiajnl-2014-002841

Lee, I. M., Shiroma, E. J., Lobelo, F., Puska, P., Blair, S. N., Katzmarzyk, P. T., \& Lancet Physical Activity Series Working Group. (2012). Effect of physical inactivity on major 
non-communicable diseases worldwide: an analysis of burden of disease and life expectancy. The Lancet, 380(9838), 219-229. doi:10.1016/S0140-6736(12)61031-9

Maher, C. A., Lewis, L. K., Ferrar, K., Marshall, S., De Bourdeaudhuij, I., \& Vandelanotte, C. (2014). Are health behavior change interventions that use online social networks effective? A systematic review. Journal of Medical Internet Research, 16(2); e40. doi:10.2196/jmir.2952

Marcus, B.H., Dubbert, P.M., Forsyth, L.H., McKenzie, T.L., Stone, E.J., Dunn, A.L., Blair, S.N. (2000). Physical activity behaviour change: issues in adoption and maintenance. Health Psychology 19, 32-41.

Marcus, B.H., Williams, D.M., Dubbert, P.M., Sallis. J.F., King, A.C., Yancey, A.K., Franklin, B.A., Buchner, D., Daniels, S.R., Claytor, R.P. (2006). Physical activity intervention studies: what we know and what we need to know: a scientific statement from the American Heart Association Council on Nutrition, Physical Activity, and Metabolism (Subcommittee on Physical Activity); Council on Cardiovascular Disease in the Young; and the Interdisciplinary Working Group on Quality of Care and Outcomes Research. Circulation 114(24), 2739-2752.

McEachan, R. R., Lawton, R. J., Jackson, C., Conner, M., \& Lunt, J. (2008). Evidence, theory and context: using intervention mapping to develop a worksite physical activity intervention. BMC Public Health, 8(1), 326. doi:10.1186/1471-2458-8-326

McIntosh, J. R. D., Jay, S., Hadden, N., \& Whittaker, P. J. (2017). Do E-health interventions improve physical activity in young people: a systematic review. Public Health, 148, 140-148. doi: /10.1016/j.puhe.2017.04.001

McVinnie, Z., Plateau, C, Stevinson, C. (2017). STRIDE: Active Fundraising - physical activity promotion through active charity events (report for Cancer Research UK). Loughborough University.

Middelkamp, J., van Rooijen, M., Wolfhagen, P., Steenbergen B. (2016). The effects of two self-regulation interventions to increase self-efficacy and group exercise behavior in fitness clubs. Journal of Sports Science and Medicine 15(2), 358-364.

Middelkamp, J., van Rooijen, M., Wolfhagen, P., Steenbergen B. (2017).The effects of a selfefficacy intervention on exercise behavior of fitness club members in 52 weeks and long-term relationships of transtheoretical model constructs. Journal of Sports Science and Medicine 16(2):163-171.

Middelweerd, A., Mollee, J. S., van der Wal, C. N., Brug, J., \& Te Velde, S. J. (2014). Apps to promote physical activity among adults: a review and content analysis. International 
Journal of Behavioral Nutrition and Physical Activity, 11(1), 97. doi:/10.1186/s12966014-0097-9

Müller-Riemenschneider, F., Reinhold, T., Nocon, M., Willich, S.N. (2008). Long-term effectiveness of interventions promoting physical activity: a systematic review. Preventive Medicine 47, 354-368. doi: 10.1016/j.ypmed.2008.07.006

Murray, J. M., Brennan, S. F., French, D. P., Patterson, C. C., Kee, F., \& Hunter, R. F. (2017). Effectiveness of physical activity interventions in achieving behaviour change maintenance in young and middle aged adults: a systematic review and meta-analysis. Social Science \& Medicine, 192; 125-133. doi:/10.1016/j.socscimed.2017.09.021

National Statistics (2018) Statistics on Obesity, Physical Activity and Diet: England 2018. NHS Digital.

Oosterveen, E., Tzelepis, F., Ashton, L., \& Hutchesson, M. J. (2017). A systematic review of eHealth behavioral interventions targeting smoking, nutrition, alcohol, physical activity and/or obesity for young adults. Preventive Medicine, 99, 197-206. doi: 10.1016/j.ypmed.2017.01.009

Pringle, A., Cooke, C., Gilson, N., Marsh, K., McKenna, J. (2010). Cost-effectiveness of interventions to improve moderate physical activity: a study in nine UK sites. Health Education Journal, 69:2, 11-24. doi: 10.1177/0017896910366790

Richards, J., Hillsdon, M., Thorogood, M., \& Foster, C. (2013). Face-to-face interventions for promoting physical activity. Cochrane Database of Systematic Reviews, (9).

Robinson, T.N. (2010) Save the world, prevent obesity: piggybacking on existing social and ideological movements. Obesity, 18 (Supp 1), 17-22. doi: 10.1038/oby.2009.427

Rundio, A., Heere, B., \& Newland, B. (2014). Cause-related versus Non cause-related sports events: differentiating endurance events through a comparison of athlete's motives. Sport Marketing Quarterly, 23(1), 17-26.

Samdal, G. B., Eide, G. E., Barth, T., Williams, G., \& Meland, E. (2017). Effective behaviour change techniques for physical activity and healthy eating in overweight and obese adults; systematic review and meta-regression analyses. International Journal of Behavioral Nutrition and Physical Activity, 14(1), 42. doi: /o10.1186/s12966-0170494-y

Schoeppe, S., Alley, S., Van Lippevelde, W., Bray, N. A., Williams, S. L., Duncan, M. J., \& Vandelanotte, C. (2016). Efficacy of interventions that use apps to improve diet, physical activity and sedentary behaviour: a systematic review. International Journal of 
Behavioral Nutrition and Physical Activity, 13(1), 127. doi:/10.1186/s12966-016-0454y

Scott, A., \& Solomon, P. J. (2003). The marketing of cause-related events: A study of participants as consumers. Journal of Nonprofit \& Public Sector Marketing, 11(2), 4366. doi: /10.1300/J054v11n02_03

Snelgrove, R., Wood, L., \& Havitz, M. E. (2013). Developing personal attachment to a physically active charity event. International Journal of Nonprofit and Voluntary Sector Marketing, 18(2), 133-140. doi:/10.1002/nvsm.1464

The Civil Society. (2017). Race for Life tops list of the biggest mass participation events in 2016. Retrieved from https://www.civilsociety.co.uk/news/139m-raised-by-top-25mass-participation-events-in-2016.html (accessed 10/12/2018)

Third Force News. (2015). Top UK charity fundraising events revealed. Retrieved from http://thirdforcenews.org.uk/tfn-news/top-uk-charity-fundraising-events-revealed (accessed 10/12/2018)

Williams, S.L., French, D.P. (2011). What are the most effective intervention techniques for changing physical activity self-efficacy and physical activity behaviour - and are they the same? Health Education Research, 26(2), 308-322. Doi: 10.1093/her/cyr005

Williams, G., Hamm, M. P., Shulhan, J., Vandermeer, B., \& Hartling, L. (2014). Social media interventions for diet and exercise behaviours: a systematic review and meta-analysis of randomised controlled trials. BMJ open, 4(2), e003926. doi:10.1136/bmjopen-2013-00

Won, D., \& Park, M. (2010). Motivating factors influencing college students' participation in charity sport events. International Journal of Sport Management and Marketing, 8(3-4), 296-321. doi: /10.1504/IJSMM.2010.03751

World Health Organisation (2010). Global recommendations on physical activity for health. Geneva, Switzerland: World Health Organisation. 
Table 1. Main findings from the Race for Life participant interviews

\section{Main findings $\quad$ Illustrative quotes}

Motivation for taking part in charity event

- To raise money for a charity that held personal relevance.

“Because I'm raising money for a charity, it encourages me to do it [Race for Life]" (Participant 01)

- To run in memory of someone or support someone with cancer.

- Inclusivity of the event (i.e., open to all abilities).

- For a physical activity challenge.

"We wanted to do something to support them, those who have survived and also in memory of those who haven 't” (Participant 14)

"We chose to walk it because that's just what we preferred and there was no pressure. We just kind of bumbled along at own pace, there was no competitive side to that at all." (Participant 13)

"It's about thinking I can still do this...for me as well it was just a bit of a challenge."

(Participant 14)

- To have fun.

"I just think it [Race for Life] is a really fun thing for everyone to do." (Participant 12)

- To assess own running ability.

"I felt that it was a way of confirming that I was really back to full fitness." (Participant 10)

\section{Outcomes from taking part in charity events}

- Increase in short-term mood.

“I was buzzing after Race for Life.” (Participant 14)

- Increase in self-efficacy for exercise.

“I think reassurance that I can still run 5k. I guess it builds confidence as well." (Participant 11) 
- Feeling of contributing towards a good cause.

\section{Barriers to physical activity}

- Lack of motivation.

- Lack of time.

- Bad weather/dark evenings.

- Child care responsibilities.

\section{Facilitators to physical activity}

- To have a training plan.

- Having a goal to work towards

- Being a fun atmosphere.

- Being able to exercise with friends and family.
"It's good for my own health and also it's my way of doing something for a very good cause and to remember my Mum." (Participant 8)

\section{"Feeling like I can't be bothered a lot of the time." (Participant 17)}

"When I went back to work I obviously don't have time." (Participant 19)

"It's now really cold and dark and I don't want to do it." (Participant 11)

"With kids you don't always... and I have always worked full-time... you don't always get the chance to do things for yourself." (Participant 14)

"I think definitely once a routine has kicked in then it is easier." (Participant 6)

"To keep running I have sort of targets and goals." (Participant 20)

“Actually you enjoy it [exercise] more when there's a fun atmosphere." (Participant 17)

"Also because just lots of friends were doing it and I thought yeah I want to do it as well." (Participant 13) 
Table 2. Matrix of change objectives for charity event participants

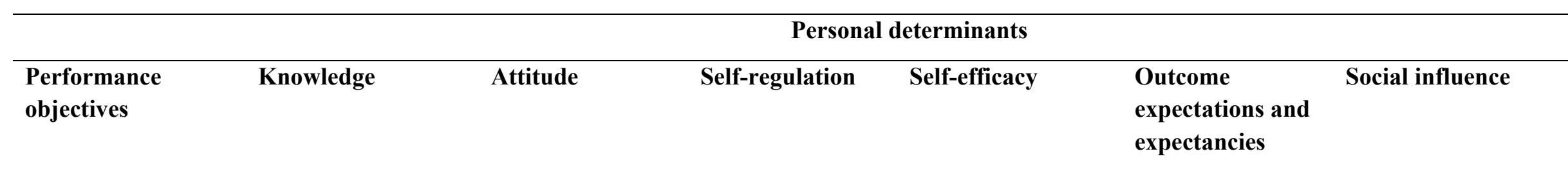

\begin{tabular}{lll}
\hline 1) Decision to & Recognise the health & Express a positive \\
train/prepare & benefits (specifically & attitude towards \\
for charity & cancer risk) of & the benefits of \\
event & deciding to & training. \\
& train/prepare. &
\end{tabular}

2) Set exercise

goals
3) Decide on a Recognise times of
weekly routine the day when
in terms of exercise is
frequency, time achievable.
and type of
exercise

Express confidence
in being able to
train/prepare.
Express confidence
in achieving goals.

Express confidence
in adhering to the
exercise routine and
being able to
engage in the type
of exercise

Express a belief

that

training/preparing

will be positively

associated with

completion of the

charity event.

Express a belief

that the goals set

will facilitate

becoming a

regular exerciser.

Express that they

value the health

benefits of regular

exercise.
Implement a

gradual increase

in the intensity of

exercise that the

plan prescribes.
Experience support for exercise from

family and friends
Family and friends to approve and respect weekly exercise routine. 
prescribed in the

regime.

4) Follow the exercise plan prior to the

event

5) Engage in a pledge to be active following

the event

6) Engage in postevent exercise
Express positive feelings towards exercising.

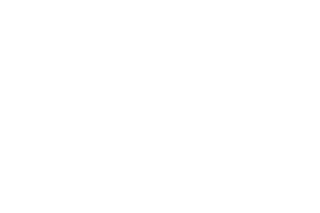

Express positive

feelings towards

post-event

exercise.

\section{Appraise exercise Express confidence \\ goals and revise if in being able to \\ necessary. follow the exercise \\ plan.}

Believe that making a pledge

will result in

greater exercise

levels.

Value the health

benefits of greater exercise levels.
Family and friends to be supportive of exercise pledge and be respectful of commitment.
Revise exercise goals to ensure regime is relevant.
Modify the exercise plan to

\section{Express confidence} in being able to
evaluate exercise plan and modify accordingly. ensure the intensity of exercise is being built upon 
7) Maintain postevent exercise

Describe the health benefits (specifically cancer-related benefits) of exercise. attitude towards maintaining exercise longterm.

\section{8) Manage}

exercise relapse
Express a belief

that continuing to

be active will

increase health

and reduce cancer

risk.

Revise the days and time exercise is being

performed to

ensure the plan is

still achievable.

Express confidence

in being able to

evaluate exercise

plan and modify

accordingly.

Revise the type of

exercise being

engaged in if

necessary (i.e.,

due to a change in

time of

year/weather/day-

time hours).
Express a belief

\section{that being a}

regular exerciser

will have a

positive

outcome(s) 
Table 3. Determinants, change methods and practical applications

\section{Determinant \\ Knowledge \\ Attitude \\ Outcome \\ expectations \& \\ expectancies} Change method Active learning

\section{Knowledge Facilitation}

Self-efficacy

Self-monitoring

Goal setting

Self-efficacy Modelling

\section{Practical application}

Design educational puzzles such as word searches and crosswords where participants can search for words to complete health and well-being related statements.

\section{Parameters for effectiveness}

Ensure content is easy to understand and evidencebased, and that tasks are achievable.
The options provided should reduce barriers to participation. available free or low-cost exercise opportunities that could appeal to individuals with different interests abilities, and circumstances.

Design an exercise planner where event participants can record 'what' exercise they plan to do, 'where' and 'when' they plan to do it, and track completion.

Provide space for event participants to set weekly exercise goals (with example goals).

Provide event participants with a choice of role models via photographs and stories. Include explicit examples of coping (e.g., "it was not easy to exercise after having a baby but I built up the amount of exercise I did each week and now I make time to exercise 3 times a week").
Monitoring should be of the behaviour itself rather than outcomes and information should be available for participants to use.

Goals should be suitably difficult but within the individual's capability. Achievement of goals requires commitment by the individual.

Role model options should take into account attention and remembrance and should be identifiable for participants. Models should demonstrate relevant skills and self-efficacy, with reinforcement of behaviour represented. Models should be based on a coping model rather than mastery. 


\section{Outcome \\ expectations and \\ expectancies}

Social influence Facilitation
Allow event participants to evaluate how they feel after the charity event and why it is important to them to become a regular exerciser.

Develop messages that encourage participants to include friends/friends when possible. Provide information on exercise opportunities that enable participation with others.
Event participants should be prompted to reflect on these responses when feeling demotivated to exercise.

Include information to enable the behaviour or reduce barriers to participation. 